\title{
LA NECESIDAD DE INCORPORAR EL CAMBIO CLIMÁTICO EN LAS POLÍTICAS DE DESARROLLO*
}

\author{
Julio García Vargas \\ Estrategia Internacional de Reducción de Desastres, Panamá \\ arquijulio59@yahoo.com
}

\section{Resumen}

El cambio climático se considera la amenaza global más importante que enfrenta la humanidad en este siglo. Originado por la desmedida emisión de gases de efecto invernadero, está ocasionando el incremento de la temperatura promedio global y, con ello, la alteración del sistema climático, además de la elevación del nivel del mar. Los países en vías de desarrollo son los que menos han contribuido a la generación del problema, pero son los que más están sufriendo sus efectos. El Perú es particularmente vulnerable a los impactos del cambio climático, pues gran parte de sus recursos y medios de producción, como la pesca, la agricultura y el transporte, son susceptibles al clima, lo que junto al reto y la oportunidad que implica planear el desarrollo sostenible en un territorio complejo en términos territoriales y poblacionales nos debe obligar a incluir en las políticas nacionales consideraciones para la reducción del riesgo de desastres y la adaptación al cambio climático. Para ello ya se cuenta con el Marco de Acción de Hyogo, aprobado por 168 países.

Palabras clave: cambio climático, adaptación, desarrollo nacional, Marco de Acción de Hyogo.

\begin{abstract}
Climate change is considered the most important global threat facing the human race in this century. Due to the excessive emission of greenhouse gases, the global average temperature is increasing thus altering climate systems and raising the sea level. Although developing countries do not contribute significantly to these emissions, they suffer most of their effects. Peru is particularly vulnerable to the impacts of climate change because most of its resources and productive factors such as fishing, agriculture and transport are climate sensitive. Combined with the challenge and opportunity of ensuring sustainable development in a complex territory in terms of land and population, this vulnerability forces us to include considerations in national policies aimed at reducing the risk of disasters and climate change adaptation. We now have the Hyogo Framework for Action approved by 168 countries.
\end{abstract}

Key words: climate change, adaptation, national development, Hyogo Framework for Action.

* Lo básico de este artículo, con modificaciones, ha sido presentado previamente en Perú Económico (2007) y en el Reporte Anual de Oxfam (2007). Esta versión se ha ampliado y se le han añadido aportes de Haris Sanahuja sobre la Estrategia Internacional de Reducción de Desastres y la implementación del Marco de Acción de Hyogo. 
El Perú es el tercer país con más riesgos climáticos a nivel mundial.

N. Brooks y N. Adger

Tyndall Center, Reino Unido, 2003

Se espera que el cambio climático va a aumentar la frecuencia y severidad de algunas amenazas naturales. A medida que nuestras ciudades y costas se vuelvan más vulnerables, estas amenazas pueden conducir a desastres de mayor alcance e impacto que los que hemos visto hasta la fecha. Tenemos, por lo tanto, una obligación social, moral y económica de aumentar la resiliencia, de aquí a 2015, como señala el Marco de Acción de Hyogo, lo cual ayudará también a alcanzar las Metas de Desarrollo del Milenio.

Ban Ki-moon Secretario General de las Naciones Unidas, 2007

\section{Introducción}

El cambio climático es reconocido como la más importante amenaza para el planeta y la sociedad humana, como un factor que alterará no solo la superficie de la tierra, sino la producción de las naciones y los medios de vida de las comunidades.

De no ser contemplado en los modos de producción e intercambio, afectará la paz mundial, la gobernabilidad de los países y, por ende, los esfuerzos por reducir la pobreza y lograr el desarrollo sostenible.
Estas circunstancias afectarán tanto el desarrollo de las comunidades locales como los propios fundamentos de la globalización por el potencial incremento de conflictos que traen y que amenazarán las estructuras de gobierno y la gobernabilidad en su conjunto.

En este contexto, el 12 de octubre de 2007 fueron proclamados como ganadores del Premio Nobel de la Paz Al Gore, ex vicepresidente de Estados Unidos, y el Panel Intergubernamental de Cambio Climático de las Naciones Unidas (IPCC por sus siglas en inglés).

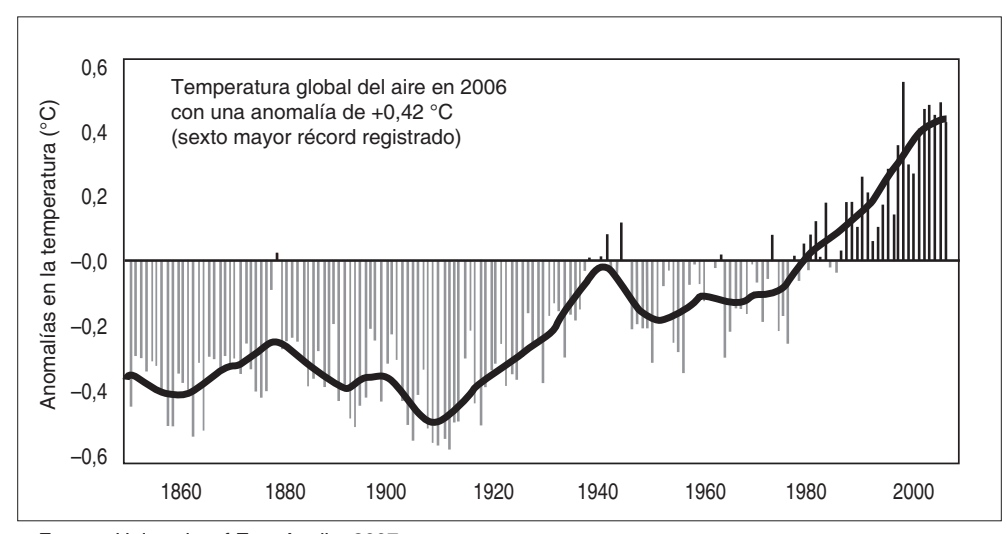

Fuente: University of East Anglia, 2007.

\section{Gráfico 1}

Temperatura y calentamiento global, 1860-2006 
El primero de ellos es reconocido por sus esfuerzos orientados a propiciar la reducción de las emisiones de gases de efecto invernadero (GEI); la realización del documental Una verdad incómoda, ganador del premio Oscar en su categoría; y una indesmayable campaña por resaltar el hecho de que evitar el cambio climático es un acto de moral y ética pública, no solo de política.

En cuanto al IPCC, se trata de un panel de más de 2500 expertos y científicos de todo el mundo que realizan reportes periódicos de evaluación de las causas y los efectos del cambio climático, basados en pruebas y observaciones de alto rigor científico. El más reciente reporte global del IPCC (2007), el cuarto, señala que el calentamiento observado se debe a la intervención humana (con un grado de confiabilidad superior a 90\%). Asimismo, que los niveles de concentración de dióxido de carbono $\left(\mathrm{CO}_{2}\right)$ exceden largamente los registros de concentraciones de los últimos 650000 años $\mathrm{y}$, probablemente, de los últimos 10 millones de años (ver gráfico 2). Igualmente, en 11 de los 12 últimos años (19952006) se han registrado los promedios más altos de temperaturas en la superficie del planeta, desde que se cuenta con registros instrumentales, y se ha tenido «picos» de temperaturas extremas en Europa, el Reino Unido y Estados Unidos.

Esto significa que la especie humana ha logrado alterar el clima mundial debido a la excesiva generación de los GEI, los cuales se han liberado en la atmósfera sin ningún tipo de restricción. Esta emisión se incrementó de manera significativa desde el inicio de la era industrial (primera mitad del siglo XIX) por causa de distintas prácticas, sobre todo de los hoy denominados países

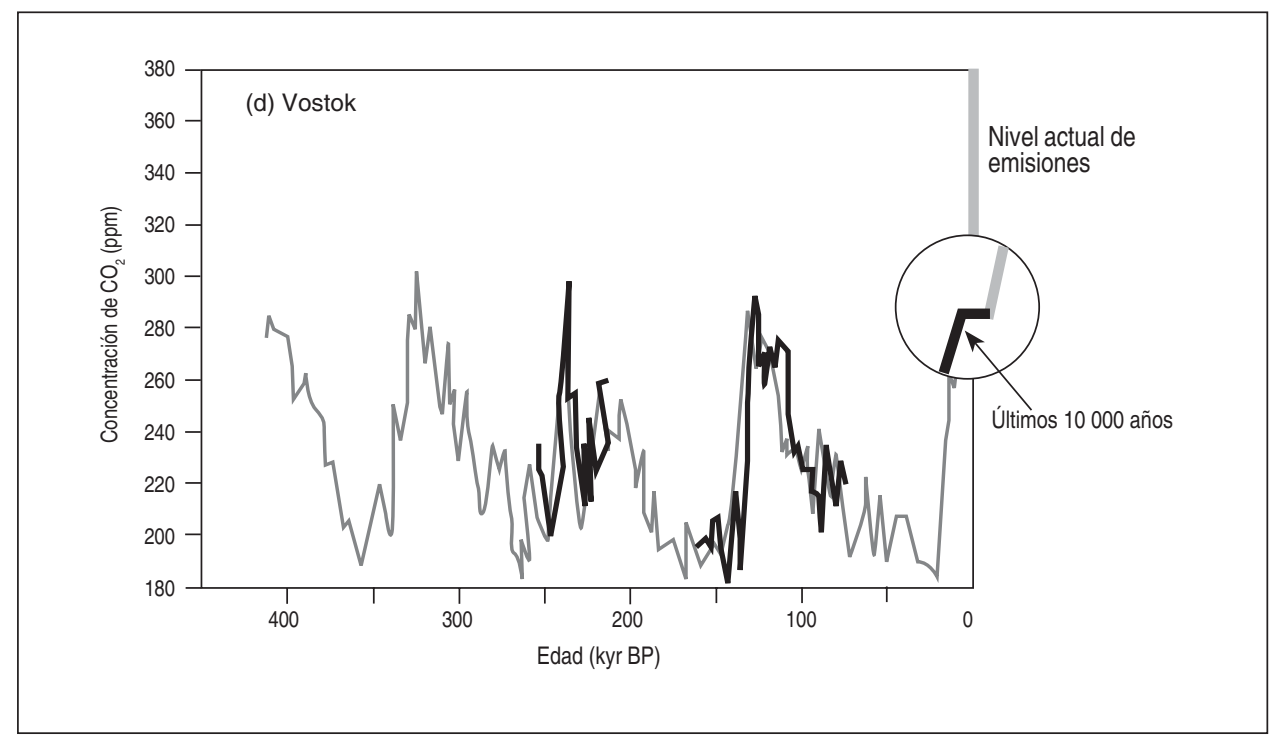

Fuente: Universidad de East Anglia (presentación en el Perú del Dr. David Viner, 2005).

\section{Gráfico 2}

Evolución nunca antes vista de los niveles de concentración de emisiones 
desarrollados, como la quema excesiva de combustibles fósiles (petróleo y derivados y carbón), la deforestación y el uso de tecnologías inapropiadas.

Hemos afectado un proceso natural necesario para la vida en la Tierra por el cual la atmósfera absorbe parte de la radiación solar que es reflejada por la superficie del planeta. Esto permite que durante la noche la tierra no se enfríe demasiado $\left(-18^{\circ} \mathrm{C}\right)$, lo que imposibilitaría la vida. Esta absorción de la radiación (retención de calor) se produce gracias a la presencia de los GEI, que siempre estuvieron presentes en nuestra atmósfera de manera natural. Los principales GEI son: dióxido de carbono $\left(\mathrm{CO}_{2}\right)$, metano $\left(\mathrm{CH}_{4}\right)$, óxido nitroso $\left(\mathrm{N}_{2} \mathrm{O}\right)$, $\mathrm{y}$ gases fluorados como perfluorocarbono
(PFC), hidrofluorocarbono (HFC) y hexafluoruro de azufre $\left(\mathrm{SF}_{6}\right)$.

Este cambio, al alterar el régimen climático e hidrológico, ocasiona mayores sequías o lluvias más intensas, con los consecuentes impactos sobre los procesos de desarrollo nacional y local. Por ejemplo, según el reporte de Global Water Partnership (2005) los niveles de precipitación en África han bajado de 16 a 25\%, lo que ha acarreado una disminución de caudales de entre 50 y $70 \%$.

Se puede observar que los peligros climáticos van en constante aumento. Como se aprecia en el gráfico 3, las pérdidas debidas a desastres naturales se han incrementado en todo el mundo, en tanto los

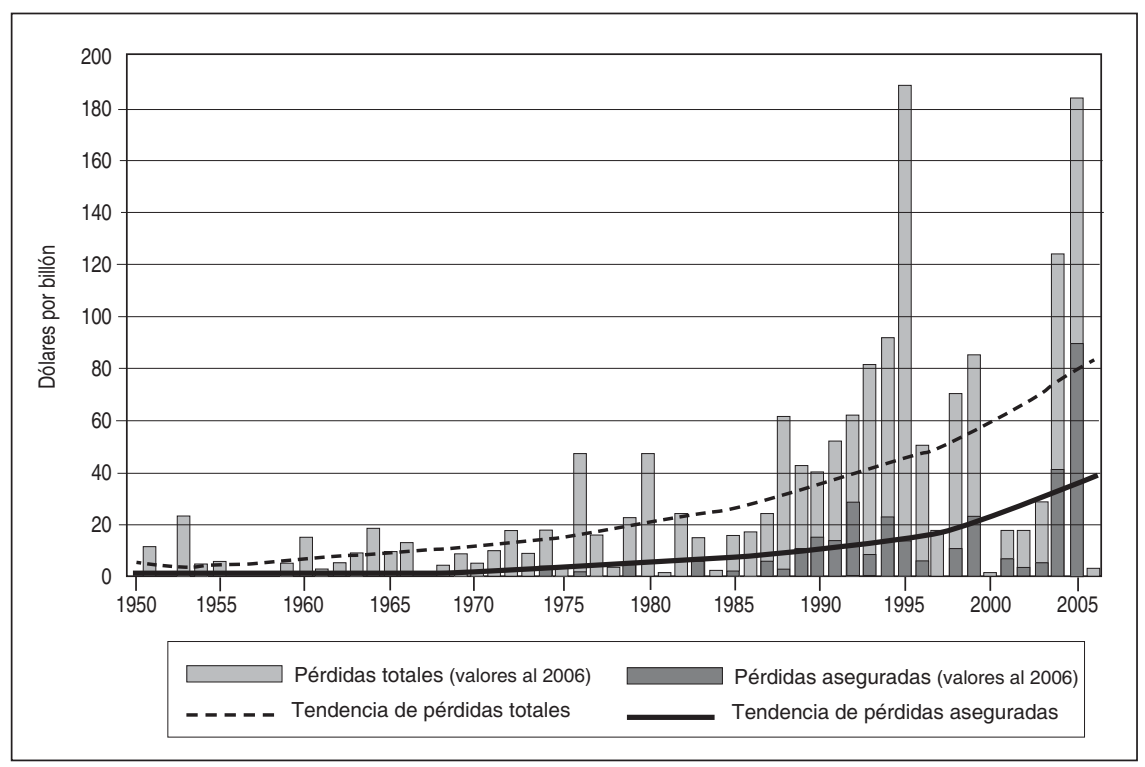

Fuente: NatCat Service, 2007.

\section{Gráfico 3}

Mayores desastres naturales, 1950-2006

Pérdidas totales y aseguradas 
montos asegurados que permitirían cubrir dichas pérdidas resultan deficitarios.

Las pérdidas económicas globales por todo tipo de desastres se han incrementado de 200 billones de dólares en la década de 1970 a cerca de 750 billones en la década de 1990. Se estima que del total de desastres al menos $74 \%$ son de origen climático. El Fenómeno El Niño (FEN) 1997-1998 generó
98 billones de dólares en pérdidas en todo el mundo (ISDR, UN, 2002).

Asimismo, el Reporte Stern ${ }^{1}$ sobre el impacto económico del cambio climático señala que se requiere de al menos $1 \%$ del PBI mundial para estabilizar los GEI en un nivel que permita el manejo de su impacto sobre el cambio climático, y que las pérdidas asociadas a estos fenómenos

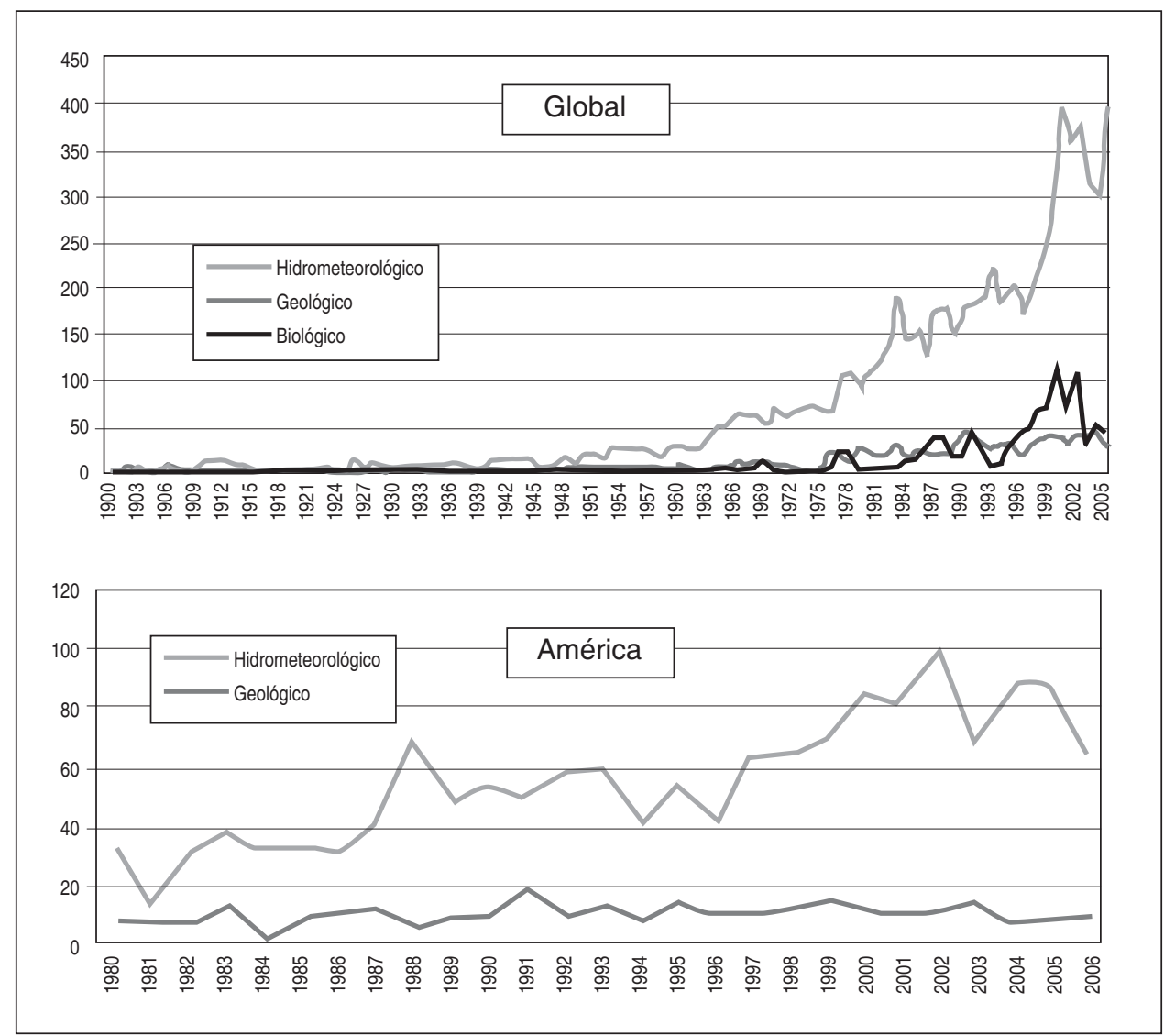

Fuente: EM-DAT, 2005.

Gráfico 4

Incremento de desastres por tipo, 1900-2005

1. Reporte encargado por el Reino Unido (a través del Primer Ministro y el Canciller) a Sir Nicholas Stern, ex jefe de economistas del Banco Mundial. 
pueden ser equivalentes a aquellas de las guerras mundiales y la Gran Depresión de la primera mitad del siglo pasado.

La tendencia global de los desastres asociados al clima se ha ido incrementando significativamente. Así, se estima que, en todo el mundo, al menos $74 \%$ de los desastres se origina en peligros asociados al clima; a pesar de que aún no se ha incluido aquellos riesgos asociados al incremento del nivel del mar.

Un reciente informe de la Iniciativa de Reporte Global (GRI por sus siglas en inglés) y KPMG (GRI / KPMG, 2007) indica, sobre la base de una encuesta a 50 empresas internacionales (ver anexo) como Citigroup, HSBC Group, Coca Cola, Shell, Samsung, Chevron, Matsushita, Mitsubishi y Petrobras, entre otras similares, que al menos $80 \%$ de ellas tienen en cuenta el cambio climático y consideran los riesgos asociados a los impactos de largo aliento de este. Además, al menos $60 \%$ ve oportunidades que estarán relacionadas con los nuevos marcos regulatorios, la oferta de nuevos servicios y la interpretación y la adecuación a las nuevas demandas (ver gráfico 5). Estas oportunidades están asociadas al mercado del carbono y a la reducción de emisiones, elementos que no se desarrollarán en este breve artículo, pues serán abordados por especialistas en el tema.

En un reporte de la Unión de Sindicatos de Europa se reconoce la existencia de más oportunidades asociadas al cambio climático, siempre y cuando se tome conciencia de las nuevas demandas, los distintos requerimientos de la industria y la reorientación de las capacidades formativas y las habilidades de trabajo de los empleados; lo que implica un compromiso serio del Estado con el otorgamiento de incentivos y nuevas oportunidades que faciliten el proceso.

\section{Por qué considerar el cambio climático en los procesos de planificación del desarrollo en el Perú}

La distribución poblacional en el territorio nacional es desigual y responde a

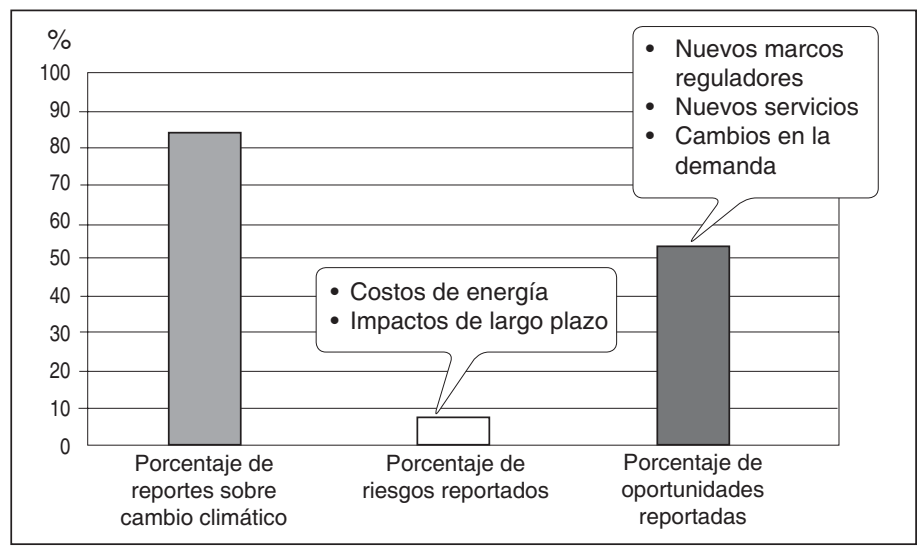

Fuente: GRI / KPMG, 2007.

Gráfico 5

Informe sobre 50 firmas internacionales: el cambio climático significa riesgos y oportunidades 


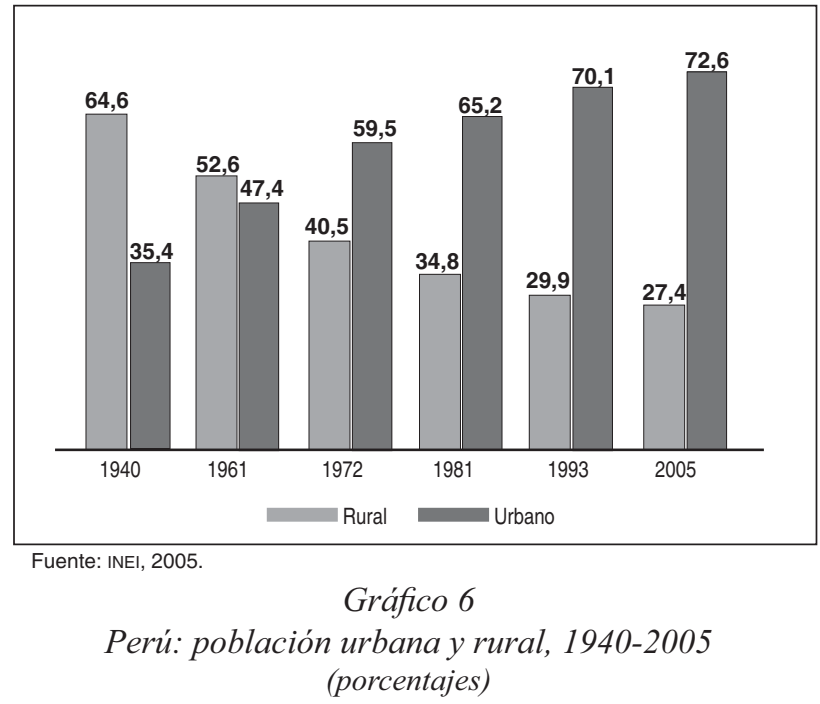

condiciones históricas e inequidades en los procesos de desarrollo económico y social marcados, entre otros factores, por una acusada centralización. Esto se refleja en el constante proceso de despoblamiento de las áreas rurales y de urbanización de la pobreza. Además, la situación se agudiza por el desplazamiento de la población a zonas costeras, principalmente áreas urbanas. Esta asimetría se acentúa al considerar el uso de los recursos naturales y, en especial, el abastecimiento y la disponibilidad de agua. Se estima que cerca de $90 \%$ de la población se ubica en $38 \%$ del territorio, el cual dispone de menos de $2 \%$ del agua del país (Inrena, 2004).

Este nivel de centralización de las actividades económicas imposibilita un crecimiento homogéneo de las oportunidades de crecimiento y desarrollo económico. En el mediano y el largo plazo, la recurrencia de fenómenos climáticos afectará la seguridad energética y alimentaria del Perú y, por tanto, pondrá en riesgo el desarrollo del país. Los fenómenos climáticos representan un serio peligro para los intereses nacionales, pues su impacto puede empañar fácilmente los avances logrados con mucho esfuerzo.

En este contexto, toda inversión (pública, privada, social, etc.) que se oriente al desarrollo debe considerar estas condiciones recurrentes, razón por la cual es imprescindible incorporar la problemática del cambio climático en los procesos de planificación del desarrollo de nuestro país. Esto nos lleva a proponer la incorporación del factor clima en la agenda de desarrollo y crecimiento descentralizado del país.

De otro lado, la enorme diversidad territorial y geomorfológica del Perú genera no solo una gran biodiversidad (estamos entre los 16 países con mayor megadiversidad), sino también múltiples pisos ecológicos (casi $84 \%$ de las zonas de vida del mundo) y climas (28 de los 35 climas existentes). Esa diversidad también se refiere a la variedad de los peligros naturales en nuestro territorio: heladas, aluviones, aludes y avalanchas, inundaciones, sequías, vientos huracanados, deslizamientos, entre otros 
peligros climáticos. Además, al estar en el Anillo de Fuego del Pacífico, tenemos alta sismicidad y vulcanismo.

Solo el FEN 1997-1998 ocasionó pérdidas por 3500 millones de dólares, 4,5\% del PBI de ese periodo (CAF, 2001), equivalente a cuatro proyectos como el de gas de Camisea. El impacto del FEN 1982-1983 se apreció en una caída del PBI que se profundizó en el periodo 1985-1990, lo que generó un PBI similar al de 1966. En la actualidad, el PBI nacional es del orden de 160 mil millones, y la pregunta es: ¿cuánto estamos dispuestos a perder por un eventual desastre asociado, sea al FEN, a heladas en el sur o a un terremoto como el de agosto de 2007 ? Sin contar el sinnúmero de «pequeños» desastres de nivel regional que no entran aún en la contabilidad de las pérdidas (ver gráfico 7).

Según la base de datos del Indeci para el periodo 1996-2005, en los últimos años las emergencias por peligros naturales han crecido en $650 \%$, al menos $72 \%$ de las cuales se originan en el clima. De esta manera, gran parte de los progresos alcanzados en aspectos como crecimiento económico, seguridad energética, infraestructura, seguridad alimentaria y gobernabilidad corren el riesgo de perderse si no se toman medidas de largo aliento para manejar la variable que amenaza el globo: el cambio climático.

Este profundizará la brecha existente entre los países, dependiendo de los niveles de desarrollo y vulnerabilidad al cambio climático de cada uno. En gran medida, el grado de afectación está en función de su estructura productiva, pues rubros como agricultura, pesca, generación de hidroelectricidad, transporte y abastecimiento de agua para consumo humano, agrícola e industrial son extremadamente sensibles a las condiciones climáticas.

Entre los principales y previsibles impactos del cambio climático están el

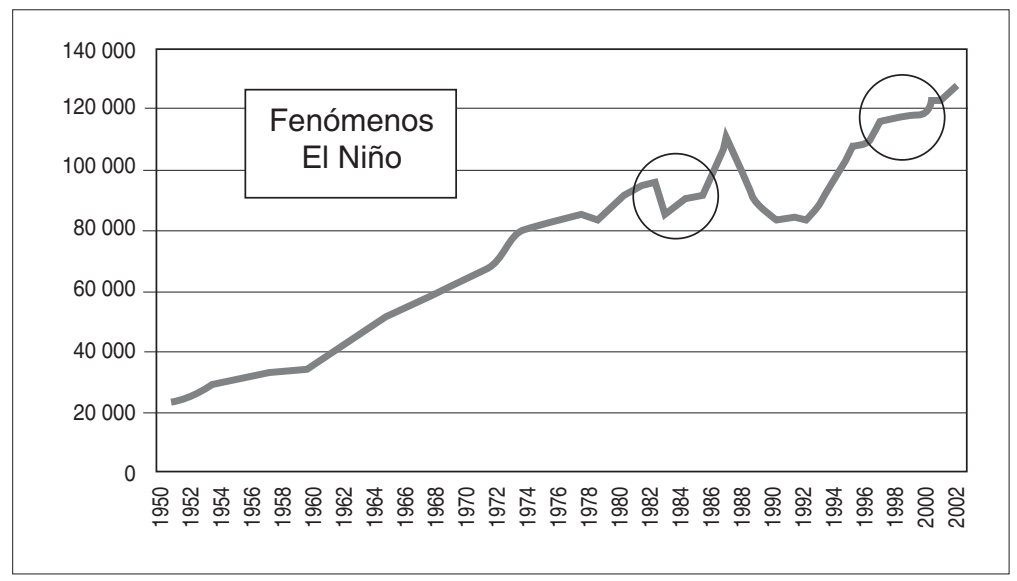

Fuente: INEI y BCR.

\section{Gráfico 7}

Perú: crecimiento del PBI per cápita y ocurrencia del FEN, 1950-2002 (millones de soles a precios de 1994) 
incremento de eventos peligrosos y extremos, como heladas, sequías y el FEN, así como el retroceso de glaciares tropicales que representan un importante recurso natural y paisajístico. A fines del siglo pasado se estimaba en $22 \%$ el retroceso de la superficie glaciar del país, con una pérdida de cerca de 7 mil millones de metros cúbicos de agua, lo que equivale al consumo de 10 años de Lima, la capital del Perú, que tiene más de ocho millones de habitantes.

A continuación se señalan algunas consideraciones que sustentan la necesidad de incorporar el cambio climático como una variable indispensable para el establecimiento de una visión estratégica del desarrollo sostenible del Perú.

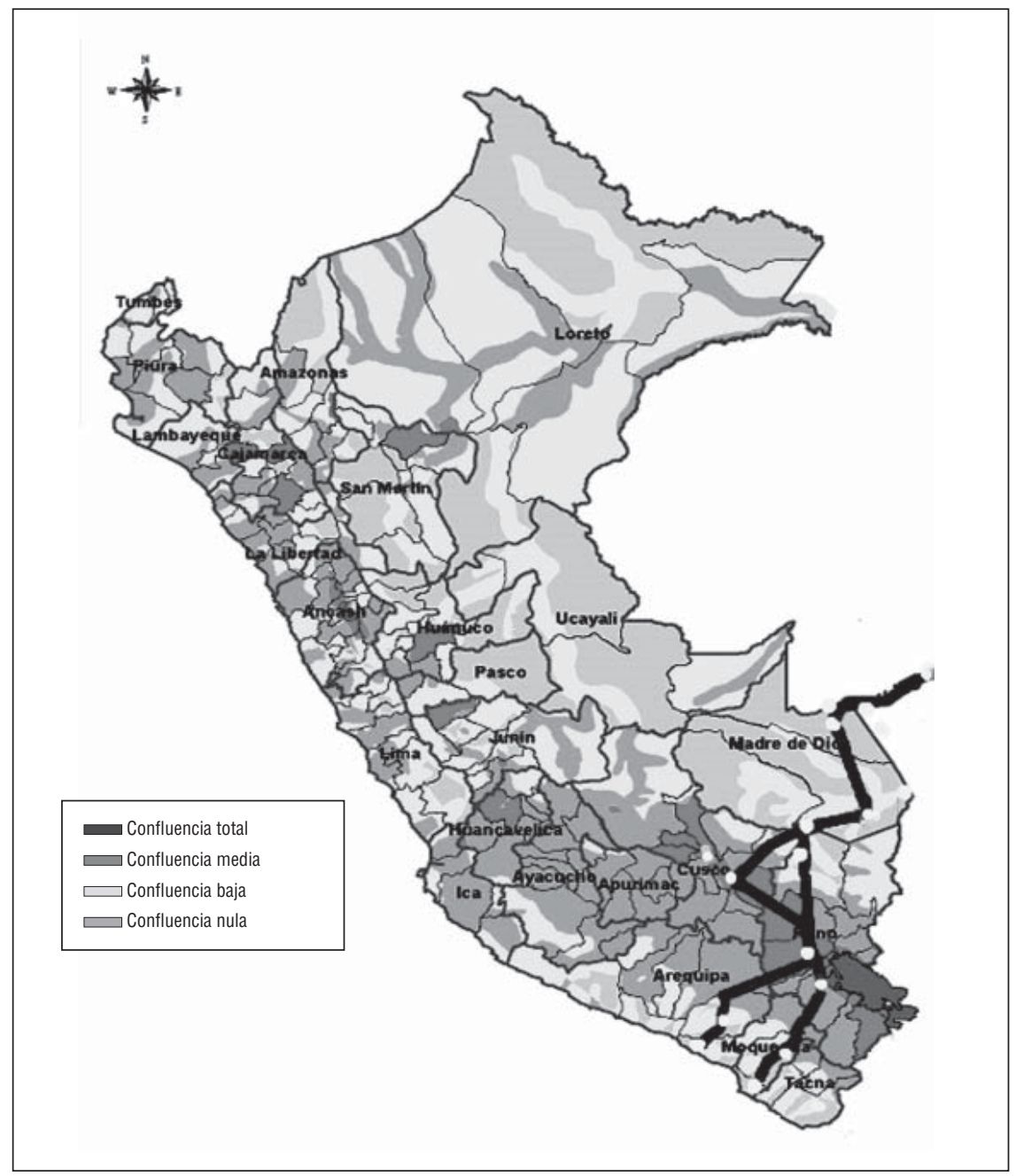

Gráfico 8

Infraestructura vial: confluencia de riesgos climáticos, pobreza y agrodiversidad 


\subsection{La carretera Interocéanica}

La mayor inversión en infraestructura vial del país aún está por desarrollarse. Este futuro activo de la sociedad peruana atravesará zonas de variado territorio y condición climática que se verían expuestas a diferentes riesgos: lluvias intensas, inundaciones, deslizamientos, nevadas y heladas, entre otros $^{2}$. La confluencia de estos riesgos climáticos con la pobreza y la agrodiversidad a lo largo de su recorrido se muestran en el gráfico 8 . Los beneficios socioeconómicos pueden no concretarse si no se toman en cuenta los factores riesgo climático y ambiental a lo largo de esta ruta.

\subsection{Cambio climático y crecimiento económico descentralizado}

Crecer no menos de 6\% al año para lograr el desarrollo es una tarea compleja y difícil. Si se compara la reducción en 4,5\% del PBI de 1998 causado por el FEN (CAF, 2001) con el impacto del proyecto de gas de Camisea (incremento potencial de 1\% en el PBI), se puede afirmar que no estamos preparados para fenómenos climáticos extremos que equivalen a perder varios megaproyectos como Camisea.

\subsection{Cambio climático y pobreza}

En el área rural del país, la pobreza total de la población llega a $70 \%$, con un $37 \%$ en pobreza extrema. La calificación del Perú como país de renta media designa una cifra agregada de carácter general pues al interior existen departamentos y provincias con niveles de pobreza comparables a los de los países menos adelantados del

2. Según datos del Indeci, $84 \%$ de las emergencias declaradas para el periodo 1995-2000 fueron de origen climático.
África subsahariana o Haití. Esto se traduce en una menor capacidad de respuesta frente al cambio climático por parte de las regiones más pobres del Perú. Estas zonas son particularmente vulnerables al cambio climático $^{3}$ debido a que la agricultura es la principal actividad económica y esta es sensible al régimen climático por ser sus sistemas agrícolas predominantemente de secano.

\subsection{Cambio climático y seguridad energética}

Aunque se pueden presentar muchos fenómenos climáticos «extremos», su frecuencia e intensidad parecen haber sido magnificadas por el calentamiento global ${ }^{4}$. Los daños generados en el subsector electricidad

3. De acuerdo con el Indeci, en el año 2004 en la región sur, debido a las heladas, se tuvo: 8 regiones y 52 provincias afectadas; daños a la población, la infraestructura, la agricultura y la ganadería; 360 mil damnificados; 66 niños fallecidos por infecciones respiratorias agudas; 250 mil animales muertos; pérdida de 216756 hectáreas de pastos naturales y 1362695 animales afectados; 34948 hectáreas de cultivos perdidas, 71964 afectadas y 111890 dejadas de sembrar. Asimismo, hubo 364323 animales afectados. Además, el año 2007 se ha exportado un porcentaje significativamente menor de mangos debido a picos de temperatura en la región norte y el año 2006 la producción lechera de Lambayeque decayó en los primeros meses del año en cerca de $30 \%$ por la elevación de la temperatura en esa zona.

4. La preocupación mundial por el problema del calentamiento global es abordada por la Convención Marco de las Naciones Unidas sobre Cambio Climático, en ese contexto, el Protocolo de Kyoto se presenta como el principal instrumento de mitigación del problema. Este protocolo ha sido aprobado por el Congreso de la República del Perú mediante la Resolución Legislativa 27824, del 6 de septiembre de 2002, y ratificado por el Decreto Supremo 080-2002-RE, del 9 de septiembre de 2002, lo que significa que su contenido tiene rango de ley nacional. 
durante el FEN 1997-1998 fueron del orden de los 464 millones de soles, y la afectación de la balanza de pagos fue de 260 millones (CAF, 2000).

\section{Estar preparados: una necesidad pública y de moral pública}

Estudios desarrollados por el Senamhi, en el marco del Programa de Cambio Climático (Proclim<www.conam.gob.pe/proclim>), estiman una mayor probabilidad de que aumente la intensidad de los futuros FEN, y se considera factible un FEN similar al de 1982-1983 en el periodo 2009-2015.

Esto implica estar preparados, pero de verdad, no solo mediante limpieza de cau$\operatorname{ces}^{5}$ que no «limpian» 15 o 20 años de decisiones mal tomadas sobre ocupación irregular, uso inadecuado del territorio y falta de definición de responsabilidades públicas en la gestión de la infraestructura económica y social y su seguridad, para que estas inversiones cumplan con los fines para los cuales fueron creadas (con gasto público, es decir, de todos los peruanos).

Este es el caso, por ejemplo, de proteger un proyecto de inversión pública-privada como Bayóvar, Piura, proyecto de larga data y aspiración regional del norte del país cuya zona de producción de fosfatos está al menos en un $70 \%$ en una zona inundable por el FEN. Por esta razón, a partir de los resultados de Proclim y esfuerzos del Gobierno Regional Piura y la GTZ, se están realizando estudios detallados que permitan una ubicación menos vulnerable de la planta y las vías de acceso a Bayóvar.

5. Palabras de un altísimo funcionario público ante una eventual presencia del FEN en las costas peruanas para el verano de 2007.
De este análisis se desprende que es ineludible un compromiso del Estado, la sociedad civil y el empresariado para alcanzar niveles de decisión política que permitan priorizar la prevención como una variable intrínseca para alcanzar el desarrollo. Con este fin tiene suma importancia contar con una base científica que permita impulsar la formulación de políticas y la toma de decisiones.

Por tanto, se considera que se debe priorizar lo siguiente:

1. Reforzamiento de los sistemas de observación regional y nacional del clima, propiciando su integración con las redes mundiales. Si bien se sabe que son los Andes los que determinan el clima de América del Sur, menos de 5\% de las estaciones hidrometeorológicas se encuentran sobre los 3 mil m.s.n.m., por lo que se conoce muy poco de la variabilidad climática en las zonas altoandinas $\mathrm{y}$, por tanto, no se pueden integrar modelos de escala global que permitan proyectar los escenarios del cambio climático en el futuro.

2. Identificación de una agenda de investigación nacional, pues lo ya señalado lleva hacia la configuración de tal agenda como herramienta y fuente de información de base para las propuestas de desarrollo nacional. Sobre este tema ha avanzado el Proclim del Consejo Nacional del Ambiente (Conam).

3. Evaluar la vulnerabilidad actual y futura del Perú. Dada la gran diversidad y la complejidad social, económica y ambiental del país, se deben seleccionar unidades territoriales en zonas específicas para evaluar el impacto y la vulnerabilidad frente a las actuales 
circunstancias climáticas (variabilidad natural) y las previsibles condiciones futuras derivadas del cambio climático global con el fin de proponer medidas de adaptación y reducción de la vulnerabilidad en el contexto del desarrollo de esas áreas. Así, estas medidas serán incorporadas en los procesos de planificación y gestión del desarrollo y, además, podrán replicarse en áreas con características similares. En estas zonas se hará evaluaciones sectoriales de acuerdo con su importancia para el desarrollo de la cuenca o región seleccionada.

4. Evaluación priorizada de ecosistemas específicos: se debe identificar áreas que por su trascendencia e impacto puedan afectar el desarrollo del país, como los ecosistemas de montaña y la disponibilidad de recursos hídricos de origen glaciar. En 35 años se han perdido 7 mil millones de metros cúbicos de agua por el deshielo de los glaciares debido al calentamiento global y, si se considera que una importante proporción de la energía del país es hidroeléctrica y parte de los grandes sistemas de irrigación de la costa del Perú utilizan aguas de origen glaciar, son muy altas las posibilidades de afectación de los sectores agrícola y energético.

5. Coordinar esfuerzos para la reducción del riesgo de desastres (RRD) impulsada por la Estrategia Internacional de Reducción de Desastres (EIRD en castellano, ISDR por sus siglas en inglés, $<$ www.eird.org $>$ ) con aquellos de adaptación al cambio climático.

En este sentido, las propuestas presentadas por diversos especialistas internacionales señalan en síntesis:
Cuando se habla del riesgo de cambio climático es importante reconocer que el punto de partida para la identificación de medidas de adaptación son las actuales condiciones de vulnerabilidad a los eventos climáticos y eventos climáticos extremos.

Mejorar las capacidades de respuesta de los gobiernos, regiones y comunidades es en muchos casos mejorar sus capacidades para hacerle frente al cambio climático (VARG, 2005).

Con miras a promover una aproximación integrada con la gestión de riegos de desastres es necesario:

- Identificar y evaluar la información, experiencias y metodologías que expertos en gestión de riesgos de desastres, cambio climático y del desarrollo puedan proporcionar, y establecer los mecanismos que permitan el intercambio de tales experiencias y conocimientos.

- Superar algunas barreras institucionales (estructurales, de gestión, de información, financieras) para facilitar la integración de experiencias, información y conocimientos de los expertos en desarrollo, cambio climático y gestión de riesgos.

6. Existe un marco de conocimientos y experiencias que son compatibles, además de la necesidad de implementar el denominado Marco de Acción de Hyogo para 2005-2015: Aumento de la Resiliencia de las Naciones y las Comunidades ante los Desastres, acordado por 168 países, entre ellos el Perú, para «la reducción considerable de las pérdidas ocasionadas por los desastres, tanto las de vidas como las de bienes 
sociales, económicos y ambientales de las comunidades y los países.

Con este fin se señalan cinco líneas de acción estratégicas:

a) Velar por que la reducción de los riesgos de desastre constituya una prioridad nacional y local dotada de una sólida base institucional de aplicación.

b) Identificar, evaluar y vigilar los riesgos de desastre y potenciar la alerta temprana.

c) Utilizar los conocimientos, las innovaciones y la educación para crear una cultura de seguridad y de resiliencia a todo nivel.

d) Reducir los factores de riesgo subyacentes (identificación del riesgo, adopción de medidas específicas, informar sobre el riesgo y movilización de recursos).

e) Fortalecer la preparación para casos de desastre a fin de asegurar una respuesta eficaz a todo nivel.

Afortunadamente, la necesidad de reducir de forma sistemática el creciente impacto de los desastres está logrando un mayor grado de reconocimiento y compromiso entre los gobiernos del mundo, en especial después del desastre que se produjo en diciembre de 2004, a raíz del tsunami en el océano Índico.

Sin embargo, la RRD representa un tema transversal y complejo, ya que requiere de un compromiso político y jurídico, al igual que el entendimiento público, el conocimiento científico, una cuidadosa planificación del desarrollo, el cumplimiento responsable de la legislación y las políticas vigentes, sistemas de alerta temprana centrados en la gente y mecanismos efectivos de preparación y respuesta. La existencia de una Plataforma Nacional multisectorial para la RRD podrá contribuir a brindar y movilizar conocimientos, destrezas y recursos para su plena incorporación a las políticas, la planificación y los programas del desarrollo.

Fundamentándose en los comités nacionales multisectoriales establecidos previamente durante el Decenio Internacional de Reducción de Desastres Naturales (DIRDN), la Secretaría de la EIRD-ONU promueve, junto con el Programa de las Naciones Unidas para el Desarrollo (PNUD) y otras agencias de las Naciones Unidas, la identificación de necesidades para el establecimiento o el ulterior desarrollo de Plataformas Nacionales para la RRD. El aspecto central de este esfuerzo es promover la importancia y la necesidad de la RRD, al igual que su plena incorporación a las políticas, la planificación y los programas de desarrollo para alcanzar el desarrollo sostenible.

Posteriormente, durante la Conferencia Mundial sobre la Reducción de Desastres, 168 gobiernos adoptaron el Marco de Acción de Hyogo para 2005-2015: Aumento de la Resiliencia de las Naciones y las Comunidades ante los Desastres (МAH). Uno de sus objetivos estratégicos es «el desarrollo y el fortalecimiento de las instituciones, mecanismos y capacidades para aumentar la resiliencia ante las amenazas». Asimismo, hace un llamado a todas las naciones para que «apoyen la creación y el fortalecimiento de mecanismos nacionales integrales, tales como las Plataformas Nacionales multisectoriales», estas se refieren a los sectores económicos convencionales y/o a las divisiones 
ministeriales o departamentales dentro del sector público, como agricultura, finanzas, salud, educación, etc., al igual que a las instituciones y los sistemas para la gestión de desastres, con el propósito de garantizar que la RRD sea una prioridad nacional y local. Este marco insta también a todos los estados a designar un mecanismo nacional para coordinar y dar seguimiento al $\mathrm{MAH}, \mathrm{y}$ comunicar cualquier información y progreso de la RRD a la Secretaría de la EIRD-ONU.

\section{Necesidad de las Plataformas Nacionales para la RRD}

La reducción del riesgo de desastres es un tema transversal y complejo, ya que requiere de un compromiso político y jurídico al igual que el entendimiento público, el conocimiento científico, una cuidadosa planificación del desarrollo, el cumplimiento responsable de la legislación y las políticas vigentes, sistemas de alerta temprana centrados en la gente y efectivos mecanismos de preparación y respuesta ante los desastres. La existencia de una
Plataforma Nacional multisectorial para la RRD puede contribuir a brindar y movilizar conocimientos, destrezas y recursos para su plena incorporación a las políticas, la planificación y los programas de desarro1lo. También puede brindar los siguientes servicios de valor agregado:

- Involucrar a los encargados de la formulación de políticas al más alto nivel a través del fomento de la RRD y su plena incorporación a las políticas, la planificación y los programas de desarrollo y asistencia humanitaria.

- Estimular la activa participación de los actores del desarrollo y los encargados de su planificación en la plena incorporación de la RRD a la agenda del desarrollo sostenible, lo que incluye los Objetivos de Desarrollo del Milenio, los Documentos sobre Estrategias para la Reducción de la Pobreza (DERP) y la Evaluación Común por País del Marco de Asistencia de las Naciones Unidas para el Desarrollo (CCA-UNDAF). Estas actividades deberán basarse en experiencias anteriores similares, como la

\section{¿Qué es una Plataforma Nacional?}

Una Plataforma Nacional para la Reducción del Riesgo de Desastres es un comité o foro compuesto por grupos mulltisectoriales que actúa en todo el país. Funge como agente promotor de la RRD en diferentes niveles, ofrece coordinación, análisis y asesoría en torno a las áreas prioritarias que requieren de acciones concertadas a través de un proceso coordinado y participativo.

Una Plataforma Nacional deberá ser el mecanismo de coordinación para lograr la plena incorporación de la RRD en las políticas, la planificación y los programas de desarrollo, en concordancia con la implementación del MAH. Su propósito deberá ser contribuir al establecimiento y el desarrollo de un amplio sistema nacional para la reducción del riesgo de desastres, de acuerdo con las características de cada país. 
integración de temas ambientales en el desarrollo.

- Ofrecer oportunidades a la sociedad civil, en especial a las organizaciones no gubernamentales (ONG) y las organizaciones comunitarias, para dialogar y contribuir al proceso de la RRD en el contexto del desarrollo local.

- Facilitar el diálogo y el establecimiento de alianzas de trabajo dentro de la comunidad internacional, lo que incluye al Sistema de la ONU y a las autoridades regionales y nacionales, especialmente mediante las Plataformas Nacionales para la RRD ya establecidas.

- Facilitar la difusión de información, el intercambio de conocimientos y la transferencia de tecnología entre los miembros de las Plataformas Nacionales para la RRD, al igual que entre las propias plataformas.

- Incrementar el acceso y los nexos de los actores de la RRD con otros entes relevantes en los ámbitos nacional, regional y mundial.

\section{A modo de corolario}

Se están desarrollando diversos esfuerzos en la arena internacional y nacional para la implementación de políticas y medidas concretas para la reducción del riesgo de desastres, la adaptación al cambio climático y la mitigación de las emisiones de gases de efecto invernadero.
El Perú ha hecho ciertos avances en algunos aspectos como la Estrategia Nacional de Cambio Climático y los resultados del Proclim. Por ejemplo, el desarrollo de la Segunda Comunicación Nacional a la Convención Marco de las Naciones Unidas sobre Cambio Climático (CMNUCC), esfuerzo liderado por el Conam con participación multiinstitucional, que aborda varios de los asuntos mencionados en este artículo.

Si bien estos avances son significativos, aún no se ha logrado traducir el cumplimiento y la aplicación de los conocimientos y las experiencias adquiridos en acciones y políticas concretas para el beneficio de las poblaciones del país, en particular de las más pobres y vulnerables, como un pilar necesario para cimentar los esfuerzos por alcanzar un desarrollo sostenible y la erradicación de la pobreza.

Sin embargo, el momento es propicio y la «masa crítica» (conocimiento público y capacidades disponibles) de apoyo a iniciativas orientadas a este fin constituyen un capital importante para motivar la decisión política y lograr un verdadero compromiso y cambio de visión de las políticas de Estado con visión de futuro, que necesariamente pasan por considerar la mitigación del cambio climático y la adaptación y la reducción del riesgo de desastres como variables intrínsecas de nuestro desarrollo, considerándolos como objetivos de ese desarrollo y no meros acompañantes o elementos del proceso de desarrollo nacional. 
Anexo. Empresas de la encuesta de evaluación de GRI / KPMG

\begin{tabular}{|c|c|}
\hline Organización & Sector \\
\hline Australia \& New Zealand Banking Group (ANZ) & Servicios financieros \\
\hline Banco Itau Holding Financeira S. A. & Servicios financieros \\
\hline Citigroup & Servicios financieros \\
\hline HSBC Group & Servicios financieros \\
\hline National Australia Bank (NAB) & Servicios financieros \\
\hline Nomura Holdings & Servicios financieros \\
\hline Royal Bank of Canada (RBC) & Servicios financieros \\
\hline Royal Bank of Scotland Group (RBS) & Servicios financieros \\
\hline Standard Bank & Servicios financieros \\
\hline TD Bank Financial Group & Servicios financieros \\
\hline Wells Fargo \& Company & Servicios financieros \\
\hline Westpac Banking Corporation & Servicios financieros \\
\hline BP & Energía (petróleo, gas y electricidad) \\
\hline Chevron Corporation & Energía (petróleo, gas y electricidad) \\
\hline Electricité de France (EDF) & Energía (petróleo, gas y electricidad) \\
\hline Eni & Energía (petróleo, gas y electricidad) \\
\hline KEPCO (Korea Electric Power Corporation) & Energía (petróleo, gas y electricidad) \\
\hline Petróleo Brasileiro (Petrobras) & Energía (petróleo, gas y electricidad) \\
\hline Sasol & Energía (petróleo, gas y electricidad) \\
\hline Shell International B. V. & Energía (petróleo, gas y electricidad) \\
\hline Suncor Energy Inc. & Energía (petróleo, gas y electricidad) \\
\hline Total & Energía (petróleo, gas y electricidad) \\
\hline Cisco Systems Inc. & Tecnología de telecomunicación e información \\
\hline Intel Corporation & Tecnología de telecomunicación e información \\
\hline Microsoft Corporation & Tecnología de telecomunicación e información \\
\hline MTN Group & Tecnología de telecomunicación e información \\
\hline NTT & Tecnología de telecomunicación e información \\
\hline Samsung SDI & Tecnología de telecomunicación e información \\
\hline Telstra & Tecnología de telecomunicación e información \\
\hline Vodafone Group PIc & Tecnología de telecomunicación e información \\
\hline Coca Cola Company & Bienes de consumo y farmacéuticos \\
\hline Johnson \& Johnson & Bienes de consumo y farmacéuticos \\
\hline Matsushita Electric Industrial Company Ltd & Bienes de consumo y farmacéuticos \\
\hline Novartis International Ag & Bienes de consumo y farmacéuticos \\
\hline Procter \& Gamble & Bienes de consumo y farmacéuticos \\
\hline Roche & Bienes de consumo y farmacéuticos \\
\hline Sony & Bienes de consumo y farmacéuticos \\
\hline Anglo Platinum Ltd & Industria y minería \\
\hline BHP Billiton & Industria y minería \\
\hline CEMEX & Industria y minería \\
\hline General Electric (GE) & Industria y minería \\
\hline Nippon Steel & Industria y minería \\
\hline POSCO & Industria y minería \\
\hline Reliance Industries Limited & Industria y minería \\
\hline Denso Corporation & Otros \\
\hline East Japan Railway (JR East) & Otros \\
\hline Hyundai Motor Company & Otros \\
\hline Mitsubishi Corporation & Otros \\
\hline Mitsubishi Estate & Otros \\
\hline Nissan & Otros \\
\hline
\end{tabular}




\section{Referencias bibliográficas}

CORPORACIÓN ANDINA DE FOMENTO (CAF). 2001. Las lecciones del Niño. Caracas: CAF.

EM-DAT: THE OFFICE OF U. S. FOREIGN DISASTER ASSISTANCE (OFDA) / CENTRE FOR RESEARCH OF THE EPIDEMIOLOGY OF DISASTERS (CRED). 2005. International Disasters Emergency Database.

GLOBAL REPORTING INITIATIVE (GRI) / KPMG. 2007. Implications of climate change in sustainability reports.

GLOBAL WATER PARTNERSHIP. < www. pwpforum.org>.

INTERNATIONAL STRATEGY FOR DISASTER REDUCTION (ISDR / EIRD). 2007. Guidelines: National platforms for disaster risk reduction. Geneve: Secretary.

- 2006. On better terms: A glance at key climate change and disaster risk reduction concepts. Geneve: Secretary.

—_. 2002. Living with risk. Geneve: Secretary.

NATCAT SERVICE MUNICH RE (Münchener Rückversicherungs-Gesellschaft). 2007. Geo Risks Research, NatCatSERVICE.
PERÚ. INSTITUTO NACIONAL DE DEFENSA CIVIL (Indeci). 1995-2000. Reportes periódicos. Lima: Indeci.

PERÚ. INSTITUTO NACIONAL DE ESTADÍSTICA E INFORMÁTICA (INEI). 2005. Censos de población. Estimaciones de población. Lima: INEI.

PERU. INSTITUTO NACIONAL DE RECURSOS NATURALES (Inrena). 2004. Comisión de Lucha contra la Desertificación. Lima: Inrena.

STERN, Nicholas et ál. 2006. Review on the Economics of climate change. London: Report to the Prime Minister and the Chancellor of the Exchequer.

UNIVERSITY OF EAST ANGLIA. 2007. Climatic Research Unit. $<$ http://www.cru. uea.ac.uk/>.

VULNERABILITY AND ADAPTATION RESOURCE GROUP (VARG). 2005. Disaster risk management in a changing climate. Kobe: The World Conference on Disaster Reduction, Outcomes Relevant to Climate Change Initiatives. 
